\title{
TRABAJADORES AGRÍCOLAS MIGRANTES EN BAJA CALIFORNIA. VINCULACIÓN CON LA MIGRACIÓN INTERNACIONAL
}

\author{
Por \\ Ma. Eugenia Anguiano Tellez*
}

\begin{abstract}
RESUMEN
En este ensayo los jornaleros agrícolas migrantes son clasificados en dos estratos: el primero, considera la migración internacional, es decir, aquéllos que se internan en Estados Unidos con el propósito de conseguir empleo; y el segundo, corresponde a la migración interna de los trabajadores agrícolas que permanecen en Baja California para laborar en los valles de Mexicali y San Quintín.

En este trabajo se sefialan las diferencias entre estos dos estratos, tanto de carácter económico como en los niveles de educación; asimismo, se comparan las características de los jornaleros agrícolas migrantes que cruzan a Estados Unidos por Mexicali y por Tijuana, según su actividad económica de procedencia y su expectativa de empleo en los Estados Unidos.
\end{abstract}

\section{ABSTRACT}

In this essay the migrant former day labourers are classified under two social strata: the first one considers international migration that is, those labourers that go into the U. S. seeking for employment; the second one refers to the internal migration of form workers that remain in Baja California to work in the San Quintin's and Mexicali's valley.

In this study the differences between both strata are appointed as in regards of their economical characters as in respect to their educational levels. Likewise, the characteristics of the migrant farm workers that cross to the United States from Tijuana and Mexicali are compared according to their financial activity source and to their job expectations in the United States.

\section{INTRODUCCIÓN}

Una parte importante del fenómeno de la emigración rural en México consiste en el traslado geográfico y condiciones de vida de los jornaleros agrícolas. Según estimaciones de la Secretaría de Agricultura y Recursos Hidráulicos (SARH), en 1980 este sector laboral, el más desprotegido

- Investigadora de Bl Colegio de la Frontera Norte, en el Departamento de Bstudios Sociales. 
económica y socialmente, constituía alrededor de 4.7 millones de personas (16 millones, considerando sus familias). ${ }^{1}$

Los jornaleros agrícolas son la fuerza de trabajo que abastece principalmente las unidades de producción más modernas del país, localizadas en regiones agrícolas que cuentan con los mejores recursos, infraestructura hidráulica y tecnología avanzada.

La agricultura del norte de México, y también del sur de Estados Unidos, son receptoras beneficiarias de estas corrientes migratorias, cíclicas y de bajo costo. Las empresas agrícolas y agroindustriales nacionales y transnacionales localizadas en Baja California, que controlan la superficie agrícola por medio de contratos de producción o de arrendamiento y concentración ilegal de tierras, se han especializado en la producción intensiva de cultivos comerciales que demandan fuerza de trabajo en forma estacionaria, con pocos trabajadores de planta. Es el caso de las empresas productoras de algodón y hortalizas ubicadas en los valles de Mexicali y San Quintín.

Por otra parte, la agricultura capitalista californiana, en donde se cosechan cerca de 250 productos agrícolas, principalmente frutas y vegetales, también ha requerido para su desarrollo de trabajadores que laboran en actividades estacionales en ciertas épocas del ciclo productivo. Esta demanda de trabajadores eventuales es cubierta por inmigrantes mexicanos que perciben bajos salarios.

Para estos jornaleros agrícolas, la posibilidad de ser incluidos en los ciclos migratorios que se internan en territorio estadounidense para trabajar, de hecho los divide en dos estratos. Los más privilegiados son los que logran cruzar la frontera, se trata de aquellos que cuentan con los recursos económicos, contactos personales, bagaje cultural e información necesarios para acceder a ese mercado laboral; el resto, cuyos recursos económi$\cos$ y redes sociales son limitados, restringen su ciclo migratorio y económico al territorio nacional, terminando sus expectativas laborales en los campos agrícolas de los estados del norte de México. La agricultura bajacaliforniana, orientada hacia el mercado internacional, cubre con ellos su demanda de fuerza de trabajo.

Para efectos del presente ensayo, denominaremos migrantes agrícolas internos a aquellos cuyo mercado laboral queda restringido al noroeste de México, mientras que consideraremos migrantes agrícolas internacionales a los que cruzan la frontera hacia California para emplearse en los campos agrícolas del vecino país.

1 Al respecto véase el informe de Narciso Acevedo, subsecretario de planeación de la Secretaría de Agricultura y Recursos Hidráulicos (1987). 
Este trabajo, de carácter exploratorio, tiene como objetivo presentar algunas de las diferencias entre ambos tipos de trabajadores agrícolas migrantes y señalar las implicaciones que tiene pertenecer a una u otra corriente migratoria, tomando como fuentes de información base los datos generados por dos estudios empíricos que se realizan en el El Colegio de la Frontera Norte (COLEF). ${ }^{2} \mathrm{Si}$ bien los datos generados en ambos trabajos de investigación no son comparables, para efectos del presente ensayo son considerados como indicativos de las tendencias y diferencias más generales entre los migrantes agrícolas internos y los internacionales.

\section{MIGRACIÓN Y MERCADOS DE TRABAJO AGRÍCOLA EN BAJA CALIFORNIA Y CALIFORNIA}

Los procesos de colonización y poblamiento de la porción norte del estado de Baja California tienen una historia reciente que data de principios de siglo. La migración intema ha sido el componente demográfico que ha determinado el crecimiento poblacional del estado. Los migrantes internos, procedentes de diversas regiones del país, constituyeron la población más numerosa durante varias décadas. Aún ahora, este estado se caracteriza por recibir corrientes migratorias; las de carácter estacional abastecen el mercado de trabajo en las actividades agrícolas, como sucede en los valles de Mexicali y San Quintín.

En Baja California existen dos zonas agrícolas de riego: la más antigua, que surge a principios del siglo, se encuentra localizada al norte del estado, en el valle de Mexicali; por otro lado, en la zona costa del muncipio de Ensenada en los años sesenta se estableció una moderna infraestructura para el desarrollo de la agricultura comercial, destinada principalmente al cultivo de hortalizas para exportación el valle de San Quintín.

Tanto en el valle de Mexicali como en San Quintín, el mercado laboral regional no permite abastecer la demanda de trabajadores agrícolas que generan los cultivos en determinadas fases del ciclo agrícola, sobre todo en la época de cosecha. El desarrollo de la agricultura en ambos valles ha dependido del abasto de trabajadores provenientes de otras regiones del país.

En el caso del desarrollo de la producción agrícola en el valle de Mexicali, al iniciar el siglo no existía un mercado laboral suficiente que permitiera cubrir la demanda de trabajadores necesarios para el desarrollo

2 Agradezco a Jorge A. Bustamante el acceso a la información generada en el proyecto Cañón Zapata, que cubre entre otras las ciudades de Tijuana y Mexicali, y a José Moreno Mena el acceso a sus hallazgos en la investigación sobre trabajadores agrícolas migrantes en el valle de Mexicali. 
de la agricultura. Dicho desarrollo estuvo estrechamente vinculado con el abasto de trabajadores provenientes de otras regiones del país. Hasta 1973, el algodón fue el producto de exportación más importante del país y el valle de Mexicali la región algodonera más productiva de México. En 1958, en el valle de Mexicali se empleaban a 30,000 jornaleros agrícolas como pizcadores de algodón (Asoc. Algodonera de B.C. 1958:136). En 1970 , la cantidad de trabajadores eventuales migratorios que laboraban en el distrito de riego del río Colorado ascendía a 45,000 (Paré, 1984:117).

En el valle de San Quintín, la actividad económica fundamental es la agricultura comercial, dedicada principalmente al cultivo de hortalizas para exportación. Actualmente, el cultivo principal es el jitomate, que ocupa el $60 \%$ de la superficie en producción. Este cultivo demanda mano de obra intensiva por temporadas. Durante la cosecha, época en que sc requiere alrededor de un $80 \%$ del total de trabajadores que participan en el ciclo productivo, laboran en los campos entre 15,000 y 25,000 jornaleros.

Actualmente, tanto en Mexicali como en San Quintín, los jornaleros agrícolas provienen de comunidades rurales empobrecidas del centro y sur del país, quienes año tras año migran solos o con sus familias para emplearse en los diferentes cultivos de la región. En general, son trabajadores agrícolas que carecen de una mínima extensión de tierra de cultivo o que poseen pequeñas parcelas de temporal, pero cuyos ingresos no son suficientes para el sostenimiento y reproducción familiar, ni aun a nivel de subsistencia. La migración no es una elección entre otras alternativas, sino una necesidad y en ocasiones la última o única opción que tienen para sobrevivir.

Una de las estrategias tradicionalmente seguidas por las unidades familiares de esas comunidades rurales consiste en enviar a uno o varios de sus miembros para que laboren durante una parte del affo en los campos agrícolas del vecino país. La diferencia salarial entre México y Estados Unidos ha resultado un incentivo considerable para los migrantes que tienen esta posibilidad.

Si bien es importante destacar el cambio que ha sufrido la demanda do trabajadores mexicanos en Estados Unidos - del sector agrícola a los serivicios y, en general, al mercado urbano industrial—, considerando a la emigración como un fenómeno social que se rige por las leyes del mercado, podemos afirmar que aún existe un sector de migrantes mexicanos que so traslada hacia los Estados Unidos en busca de mercados de trabajo agrícola más favorables. Al respecto Francisco Alba señala:

“... ya hace tiempo que la demanda por este tipo de trabajador ha dejado de ser dinámica, la demanda existe, pero se mantiene 
estacionaria ... la agricultura ha venido ocupando una proporción decreciente de la población activa total y un número de activos casi constante en términos absolutos. Este desarrollo obviamente ha estado afectando la demanda de este tipo de trabajadores migrantes, no cancelándola, pero desde luego no dinamizándola." (Alba, 1986:3).

De acuerdo con los datos de la Entrevista Continua del Proyecto Cañón Zapata, que se realiza en los principales puntos fronterizos de cruce de inmigrantes ilegales mexicanos a los Estados Unidos, en las ciudades de Tijuana y Mexicali aún se observa una proporción de migrantes con expectativas de empleo en el sector agrícola.

En el caso de Tijuana, de un total de 3,859 entrevistas realizadas de junio de 1987 a octubre de 1988, 1,597 inmigrantes (41.3\%) tenían como empleo de procedencia el sector agrícola, mientras que 1,147 (29.7\%) tenían la expectativa de emplearse en los Estados Unidos en ese mismo sector. Del total de los inmigrantes entrevistados, aquellos que tenían como empleo de procedencia el sector agrícola o bien tenían como expectativa el emplearse en ese sector en los Estados Unidos, es decir, que reunían alguna de estas dos características, ascendió a 1,961 casos, que representan el $50.81 \%$ del total.

En el caso de Mexicali, de 1,705 entrevistas levantadas entre octubre de 1987 y junio de 1988, observamos que 985 inmigrantes $(57.77 \%$ ) pertenecían al conjunto de trabajadores provenientes del sector agrícola 0 con expectativas de empleo en ese mismo sector en el lugar de destino. Los casos cuyo empleo de procedencia era el sector agrícola ascendieron a $851(49.9 \%)$, mientras que aquellos que declararon tener como expectativa de empleo este mismo sector en los Estados Unidos sumaron 902 (52.9\%). Comparativamente tendríamos la siguiente situación:

$\begin{array}{lcc}\begin{array}{l}\text { Ciudad } \\ \text { cruce }\end{array} & \begin{array}{c}\text { Empleo de } \\ \text { procedencia } \\ \text { (sector agrícola) }\end{array} & \begin{array}{c}\text { Expectativa de } \\ \text { empleo } \\ \text { (sector agrícola) }\end{array} \\ \text { Tijuana } & 41.3 \% & 29.7 \% \\ \text { Mexicali } & 49.9 \% & 52.9 \%\end{array}$

Las diferencias entre los datos de Tijuana y Mexicali, en términos muy generales, podrían indicarnos la decisión de los inmigrantes mexicanos de cruzar por una u otra ciudad en relación a la ubicación y rama de actividad económica de los mercados laborales del otro lado de la frontera. Considerando el flujo regional por actividad económica, tendríamos que las corrientes migratorias de trabajadores agrícolas no pasan principalmente 
por Tijuana. Los inmigrantes que cruzan por Tijuana esperan ubicarse más bien en los mercados del sector industrial y de servicios, aun cuando su empleo de procedencia sea de tipo agrícola; mientras que en Mexicali, un pequeño sector de procedencia laboral no agrícola espera ubicarse precisamente en ese sector. En el caso de Mexicali, la vecindad con el Valle Imperial es un elemento a considerar en la diferencia de las expectativas de empleo agrícola declaradas por los entrevistados en las dos ciudades.

\section{CORRIENTES MIGRATORIAS INTERNA E INTERNACIONAL: TENDENCIAS Y DIFRENCIAS}

En la actividad agrícola que se desarrolla en las grandes empresas capitalistas de Baja California y California, las corrientes migratorias se encuentran vinculadas a las labores agrícolas y ciclos de los diferentes cultivos; labores y ciclos marcan el itinerario a seguir por los trabajadores que en ellas se emplean.

Existen otros elementos que definen ese itinerario, como por ejemplo: la calificación del trabajador y los recursos con que cuenta para trasladarse a los distintos mercados laborales. Como seffalamos inicialmente, la diferencia entre los trabajadores agrícolas que migran a Baja California y los que se trasladan a los campos de California es básicamente una diferencia relacionada con los recursos económicos con los que cuentan. Asimismo la tradición migratoria de los lugares de origen y las redes familiares tienen un peso importante. $\mathrm{Al}$ respecto, los datos obtenidos en las investigaciones realizadas en el COLEF sobre migración interna e internacional a Baja California y California nos permiten señalar las siguientes diferencias y tendencias generales.

\section{Diferencia salarial}

En los campos agrícolas de San Quintín el salario mínimo oscila entre 9,335 y 11,335 pesos diarios, en Mexicali el rango varía entre 7,980 y 12 mil pesos diarios. En el Valle Imperial el salario mínimo por hora es de 3.75 dólares; si consideramos una jornada promedio de ocho horas, el salario diario sería de 30 dólares, que a tipo de cambio de 2,300 pesos por dólar equivaldría a 69 mil pesos diarios. Esta diferencia nos indica la ventaja de trabajar en los campos agrícolas del vecino país; sin embargo, no todos los trabajadores agrícolas migrantes tienen acceso a ese mercado. Cruzar a California implica una serie de gastos que un salario mínimo agrícola nacional no permite cubrir. 
Por otra parte, el bajo costo de la fuerza de trabajo agrícola de este lado de la frontera, explica en parte la presencia de las considerables inversiones de las companías agroindustriales norteamericanas, y también nacionales, en los campos agrícolas de Baja California. ${ }^{3}$

\section{Ruta migratoria}

Respecto a las diferencias en torno a la ruta migratoria que siguen los trabajadores agrícolas, es importante considerar dos tipos de variables: la económica, o recursos necesarios para acceder a un destino nacional o internacional; y la cultural, que conlleva la tradición en el lugar de origen de migrar y las redes familiares (en sentido amplio) que facilitan esa migración. Aun en el caso de los migrantes internos que laboran por temporadas en Baja California, las variables económica y cultural imprimen diferencias entre aquellos que llegan a Mexicali o San Quintín.

Los lazos personales y familiares son puentes de integración entre los migrantes, vividos con mayor intensidad precisamente por estar fuera del espacio geográfico de la comunidad. Entre otros, estos elementos determinan la dirección del movimiento migratorio y el lugar de empleo. Es el caso de una gran parte de los trabajadores agrícolas provenientes del estado de Oaxaca, cuyas redes familiares han definido su empleo de destino en el valle de San Quintín y no en el de Mexicali (o bien la preferencia por determinado patrón contratante y no por otro, o el lugar para levantar un techo de cartón, etc.). Esta información, y la posibilidad de utilizarla con exito dependen de esa experiencia colectiva de la comunidad (Anguiano, 1986).

La ruta migratoria de los trabajadores que llegan a San Quintín inicia en los meses de enero y febrero en los campos agrícolas de Sinaloa, posteriormente se dirigen hacia el valle del Yaqui en Sonora, donde una vez concluidos los trabajos del ciclo agrícola, algunos retornarán a sus comunidades de origen para trabajar sus tierras, mientras que otros serán reclutados para trabajar durante los meses de junio a noviembre en los campos de San Quintín.

Por su parte, la mayoría de los trabajadores que llegan al valle de Mexicali recorren una ruta que inicia en los estados de Nayarit o Sinaloa en los meses de diciembre a mayo, continúa hacia Sonora durante junio y

3. A las ventajas económicas en términos de costos de producción habría que agregar las condiciones y significativas diferencias de la organización laboral de los trabajadores en uno y otro lado de la frontera. Pensemos, por ejemplo, en la capacidad de convocatoria a la población que ha logrado la United Farm Workers (UFW) en los Estados Unidos. 
agosto para finalizar en Mexicali durante los meses de agosto a diciembre. Estos trabajadores, en su mayoría hombres solos (sin familia o redes familiares equivalentes) que vienen "siguiendo las pizcas", siempre saben dónde habrá trabajo la siguiente temporada.

Si bien ellos no establecen amplias redes familiares, ni se encuentran vinculados a comunidades de origen o residencia a los cuales regresar durante algunos meses del año, como miembros de un mismo flujo migratorio regional (la llamada corriente del noroeste) comparten información que les permite saber en qué cultivos y en qué épocas del año habrá trabajo en estos estados que recorren de manera circular y permanente. Las redes sociales que acompañan y fortalecen el proceso migratorio les imprimen características de colectividad y no de trabajadores aislados.

Las redes familiares implican también diferencias en la generación y distribución del ingreso y, en general, en las estrategias de reproducción individuales, familiares y aun comunales. Asimismo, conservar un lugar de origen, procedencia o residencia al cual regresar en cierta época del affo, implica la posibilidad de no laborar durante ese periodo. Para los jornaleros migrantes que se emplean en el valle de Mexicali, el lugar de nacimiento se ha convertido en algo circunstancial, mientras que la mayoría de los trabajadores agrícolas que laboran en San Quintín aún conservan un lugar al cual aspiran regresar cuando el término de la temporada lo permita.

Los migrantes internacionales también tienen relaciones familiares y una cultura que ha determinado no sólo los destinos, sino también lo que se ha llamado la tradición de migrar. Al respecto, los datos de la Encuesta Continua del Proyecto Cañón Zapata nos indican la recurrencia de los lugares de origen tradicionales de los que provienen esos migrantes. Para el caso de Tijuana se encontraron entre los primeros diez lugares de origen más frecuentes a los estados de: Michoacán, Oaxaca, Jalisco, Guerrero, Guanajuato, Sinaloa, Nayarit, Puebla, Distrito Federal y Colima. En el caso de Mexicali se observa el siguiente orden: Michoacán y Sinaloa, Guanajuato, Jalisco, Nayarit, Zacatecas, Oaxaca, Sonora, Guerrero, Morelos.

Michoacán, Jalisco y Guanajuato son estados de gran tradición en su aportación de trabajadores migrantes a diversos mercados laborares en los Estados Unidos. Por otra parte, Sinaloa, Nayarit, Oaxaca y Sonora se encuentan integrados a lo que podríamos denominar el flujo regional de migración del Pacífico. ${ }^{4}$

$4 \mathrm{Al}$ respecto, en la investigación realizada en el proyecto Cañón Zapata se han sefialado flujos regionales de migración a partir de la vinculación entre los lugares de procedencia de los trabajadores mexicanos y los correspondientes destinos laborales en los Estados Unidos. 
La posibilidad de trasladarse del lugar de origen o residencia al destino laboral en los Estados Unidos implica contar con los recursos económicos suficientes para cubrir los costos del viaje, cruce, estancia, pago de agente migratorio ilegal y demás. La decisión de cruzar, a pesar de la atractiva diferencia salarial del mercado laboral agrícola en California, es una opción que tiene en cuenta principalmente razones económicas. La pertenencia a una u otra corriente migratoria, dentro y fuera del país, lo es también a un estrato social diferente entre los trabajadores agrícolas.

\section{Niveles de educación formal: calificación del trabajador}

De acuerdo con las entrevistas realizadas por José Moreno Mena en su investigación sobre trabajadores agrícolas migrantes en el valle de Mexicali, la escolaridad promedio entre ellos no excede el segundo grado de educación primaria. En el valle de San Quintín, el acceso a la educación primaria para los migrantes (niños, jóvenes y adultos) no solamente está restringido por la falta de escuelas en los campamentos en que habitan las familias de los trabajadores (Hernández, 1988), sino también por la continua migración familiar - que no coincide con la calendarización escolar formal, sino con la demanda de trabajadores en ciertas épocas del año en diferentes mercados laborales-y por la imperiosa necesidad de la unidad familiar de integrar al mayor número de sus miembros en actividades productivas, los cuales son factores que determinan un promedio de escolaridad que muy excepcionalmente alcanza el tercer grado de educación primaria.

La tendencia observada en los migrantes internacionales respecto al grado promedio de educación formal es diferente. De los datos generados en la investigación del Proyecto Cañón Zapata en las ciudades de Tijuana y Mexicali, seleccionamos las variables relacionadas con los grados de escolaridad declarados por aquellos migrantes cuyo empleo en el lugar de origen, o expectativa de empleo en el lugar de destino, se encontraba en el sector agrícola.

En el caso de Tijuana, un $49 \%$ de esos migrantes cursó hasta sexto grado de primaria y un $14 \%$ hasta tercer grado de secundaria. En el caso de Mexicali, un $46.8 \%$ cursó los seis grados del nivel primaria, y un $19 \%$ los tres años de secundaria. Los migrantes internacionales en ambas ciudades alcanzaron promedios bastante similares en la educación formal, comparativamente por encima de la media nacional. Esto significa que la migración de mexicanos a los Estados Unidos no sólo ha cambiado en términos de la composición laboral de los mercados de destino (del sector agrícola a los servicios y la industria), sino también en la calificación de 
los trabajadores que aún se emplean en sectores agrícolas; aunque tambic" podría ser un indicador del impacto o la fuerza con que la crisis económica del país ha afectado a sectores sociales con grados de escolaridad más alton que el promedio nacional, quienes han tenido que migrar para contratarso (según el conjunto de nuestra selección de datos) incluso en empleon agrícolas en los Estados Unidos.

Además del acceso que los migrantes internacionales han tenido a lı educación formal, han podido concluir no solamente años escolares, sino ciclos completos de educación básica y aun de educación media. Esto refleja diferencias importantes en relación con los migrantes internos: la posibilidad de acceso a altos grados de educación formal puede traducirse en la posibilidad, para la unidad familiar, de reproducirse sin la necesaria y pronta integración de algunos de sus miembros a la vida productiva.

\section{ALGUNAS CONSIDERACIONES FINALES}

Las diferencias que existen entre las corrientes migratorias del país, relacionadas con los mercados de empleo urbanos o agrícolas de los que provienen y a los que se trasladan los trabajadores, así como los lugares de destino nacionales o internacional (en este caso Estados Unidos), nos permiten pensar en diferencias sociales importantes que los colocan en distintos estratos e implican diferencias significativas en sus condiciones generales de vida.

Para nuestro caso, en términos de la posible elaboración de una tipología que nos permitiera comparar las diferencias entre los migrantes agrícolas que se trasladan a Baja California (México) y California(Estados Unidos), una primera división fue establecida a partir del mercado laboral urbano o agrícola de procedencia o destino, dejando de lado el primero. Para el análisis, esto implicó restringir la población a aquellos migrantes que provienen de $\longrightarrow$ se trasladan a - mercados laborales agrícolas, e implica también considerar en ésta una división importante entre ambos tipos de migrantes.

Entre los migrantes agrícolas econtramos también diferencias significativas, marcadas no únicamente por el destino nacional o internacional, sino también por la región en que se ubican los mercados laborales en uno y otro lado de la frontera. El acceso a los distintos mercados regionales no sólo implica diferencias económicas entre estos trabajadores, sino también diferencias de carácter social y cultural que incluyen, entre otras, tradiciones migratorias vinculadas con las comunidades de origen y relaciones familiares y laborales. En el caso que nos ocupa, encontramos diferencias importantes entre los migrantes internos que tiene como mercado laboral 
el valle de Mexicali o los campos agrícolas de San Quintín; y entre los migrantes internacionales aquéllos que tiene como punto de cruce las ciudades de Tijuana o Mexicali.

Finalmente, las variables consideradas además de permitirnos señalar concretamente esas diferencias, nos darían la posibilidad de elaborar una interesante tipología de estos migrantes agrícolas. 


\section{BIBLIOGRAFÍA}

ACEVEDO Valenzuela, Narciso. 1987. "Condiciones de vida y trabajo de los jornaleros agrícolas en México", Ponencia presentada en la Reunión Sobre Jornaleros Agrícolas en México y Estados Unidos, Tijuana, COLEF.

ALBA, Francisco. 1986. "Oferta y demanda de trabajadores migratorios en México y Estdos Unidos: una perspectiva global”. Ponencia presentada en el Seminario Interdependencia entre los Mercados de Trabajo de México y Estados Unidos. El Colegio de México.

ANGUIANO, Ma. Eugenia. 1986. "Los mixtecos en Baja California". En: México Indígena. México, Instituto Nacional Indigenista. No. 13, año 2.

ASOCIACIÓN ALGODONERA DE BAJA CALIFORNIA Y SANLUIS RÍO COLORADO. 1958. Ponenecia presentada ante el CEPES. Mexicali, B.C.

HERNÁNDEZ, Alberto, et al. 1988. "Informe de la reunión regional con migrantes indígenas". San Quintín, Comsión de desarrollo de zonas indígenas.

PARÉ, Luisa. 1984. El Proletariado agrícola en México. México, Siglo XXI Eds. 